\title{
Clinical significance of vasculogenic mimicry in human gliomas
}

\author{
Xiao-mei Liu $\cdot$ Qing-ping Zhang $\cdot$ Yong-gao Mu $\cdot$ \\ Xiang-hen Zhang $\cdot$ Ke Sai $\cdot$ Jesse Chung-Sean Pang • \\ Ho-Keung Ng $\cdot$ Zhong-ping Chen
}

Received: 6 December 2010/Accepted: 6 April 2011/Published online: 30 April 2011

(c) The Author(s) 2011. This article is published with open access at Springerlink.com

\begin{abstract}
Vasculogenic mimicry (VM) is known as nonendothelial tumor cell-lined microvascular channels in aggressive tumors. We have previously found the presence of VM in high-grade gliomas. In this study, we aimed to identify VM patterns in gliomas and to explore their clinical significance. Tumor samples as well as their detailed clinical/prognostic data were collected from 101 patients. Vasculogenic mimicry in the glioma samples was determined by dual staining for endothelial marker CD34 and periodic acid-Schiff (PAS). Tumor samples were also immunohistochemically stained for Ki-67, VEGF, COX-2 and MMP-9. The association between VM and the clinical characteristics of the patients were analyzed. A KaplanMeier survival analysis and log-rank tests were performed to compare survival times of the patients. Vasculogenic mimicry was present in 13 out of 101 samples. The higher grade gliomas had a higher incidence of VM than that of lower grade gliomas $(P=0.006)$. Vasculogenic mimicry channels were associated with the expression of COX-2 and MMP-9 $(P<0.05)$. While there was no association between the existence of VM and the sex, age and preoperative epilepsy of the patients, or expression of Ki-67 and VEGF. However, patients with VM-positive gliomas survived a shorter period of time than those with VM negative gliomas $(P=0.027)$. Interestingly, in high-grade gliomas,
\end{abstract}

X. Liu $\cdot$ Q. Zhang $\cdot$ Y. Mu $\cdot$ X. Zhang $\cdot$ K. Sai $\cdot$ Z. Chen $(\square)$

State Key Laboratory of Oncology in South China

and Department of Neurosurgery/Neuro-Oncology, Cancer

Center, Sun Yat-sen University, 651 Dongfeng Road East,

Guangzhou 510060, People's Republic of China

e-mail: chenzhp@sysucc.org.cn

J. C.-S. Pang · H.-K. Ng

Department of Anatomical and Cellular Pathology, The Chinese

University of Hong Kong, Hong Kong, China the level of microvascular density was lower in VM positive tumors than those VM negative tumors $(P=0.039)$. Our results suggest that VM channels in gliomas correlate with increasing malignancy and higher aggressiveness, and may provide a complementation to the tumor's blood supply, especially in less vascularized regions, which may aid in the identification of glioma patients with a poorer prognosis.

Keywords Angiogenesis - Glioma $\cdot$ Microvascular density $\cdot$ Prognosis · Vasculogenic mimicry

\section{Introduction}

Glioma is the most common primary brain tumor, accounting for about $50 \%$ of all central nervous system neoplasms. The prognosis for glioma patients is not satisfactory despite multimodality management with surgery, radiation, and chemotherapy. The median survival time for patients with glioblastoma multiforme (GBM), the most malignant glioma, is still only 1 year [1]. Prolonging survival in glioma patients remains a challenge in the field of neuro-oncology.

In 1999, Maniotis et al. [2] reported that highly aggressive uveal melanomas could form blood vessels by tumor cells instead of endothelial cells, and named this phenomenon of tumor vascularization as vasculogenic mimicry (VM). Since then, VM has been described in several malignant tumors including prostatic carcinoma [3], inflammatory and ductal breast carcinoma [4], ovarian carcinoma [5], rhabdomyosarcoma [6] and osteosarcoma [7]. Vasculogenic mimicry has also been implicated in invasion and metastasis, and is associated with poor prognosis in hepatocellular carcinoma [8] and gastrointestinal stromal tumors [9]. The presence of VM is associated 
with more aggressive tumor biology and increased tumorrelated mortality [10].

There is limited data regarding VM in human gliomas. We previously reported the presence of VM in human gliomas [11]. The aim of this study was to perform a systematic evaluation of VM in gliomas of different grades and to correlate such findings with clinicopathological parameters.

\section{Materials and methods}

Patients and tissue samples

One hundred and one patients pathologically diagnosed with gliomas from 2000 to 2006 at Cancer Center of Sun Yat-sen University were analyzed. All patients received craniotomy for tumor resection and had detailed clinical follow-up data. Tumor sections were reviewed by two neuropathologists to verify diagnosis according to World Health Organization (WHO) 2007 classification standards for central nervous system tumors [12]. Only astrocytic tumors were included in this study. The series consisted of 6 grade I astrocytomas, 35 grade II astrocytomas, 39 grade III anaplastic astrocytomas (AA), and 21 grade IV glioblastoma multiformes (GBM). Patients with mixed gliomas were excluded from the study.

\section{CD34-PAS dual staining}

Vasculogenic mimicry was detected by CD34-PAS dual staining as described previously [11]. Briefly, standard immunohistochemical staining was performed on $5-\mu$ formalin-fixed paraffin-embedded tumor sections, for CD34 (1:200, polyclonal antibody; Santa Cruz Biotechnology), followed by immunodetection using the EnVision ${ }^{\mathrm{TM}}+$ System (Peroxidase Kit; Santa Cruz Biotechnology). The slides were then rinsed with distilled water for $5 \mathrm{~min}$, incubated with periodic acid-Schiff (PAS) for $15 \mathrm{~min}$, counterstained with Mayer's hematoxylin for $1 \mathrm{~min}$, and viewed under a light microscope to detect CD34 and PAS signals. The whole section was examined for the presence of VM (CD34-negative and PAS-positive vessels) by three independent observers without knowledge of patient outcome. Microvascular density (MVD) was evaluated by counting CD34-positive cells in 10 randomly selected fields at high magnification and the average counts were then calculated. Adult liver samples were used as controls.

Immunohistochemical studies

Formalin-fixed paraffin-embedded specimens were analyzed by immunohistochemistry with antibodies of glial fibrillary acidic protein (GFAP) (1:500; Dakocytomation, Glostrup, Denmark), Ki-67, VEGF, COX-2 and MMP-9 (1:200; Santa Cruz Biotechnology) according to manufactory instruction. Immunodetection was performed using the EnVision $^{\mathrm{TM}}+$ System (Peroxidase Kit; Santa Cruz Biotechnology). The slides were rinsed with distilled water for $5 \mathrm{~min}$, counterstained with Mayer's hematoxylin for $1 \mathrm{~min}$, dehydrated through an alcohol gradient and cover-slipped. Tissues from five normal brains (from decompression surgery for head injury patients) served as controls.

Nuclear staining for $\mathrm{Ki}-67$ and cytoplasmic staining for VEGF, COX-2 and MMP-9 were considered positive. The degree of immunostaining was scored separately by two independent investigators, who were blinded to the histopathologic features. The scoring of Ki-67 was based on percent positivity [13]. Tumors were classified as 0 for negative, 1 for $\leq 10 \%, 2$ for $10-25 \%$ and 3 for $\geq 25 \%$ staining. The intensity of staining was graded as 0 for absent immunoreactivity, 1 for weak, 2 for moderate, and 3 for intense positivity. The staining intensity index of VEGF, COX-2 and MMP-9 was determined by multiplying the proportion of positively stained tumor cells and the intensity of staining.

\section{Statistical analysis}

The statistical analysis was performed with SPSS v.13.0 for Windows. Associations between VM and clinical data were determined by the Pearson's $\chi^{2}$ test. A Kaplan-Meier survival analysis and log-rank tests were performed to compare survival times of patients between the VM and non-VM groups. The multivariate survival analysis was calculated according to the Cox proportional hazards model. The threshold for statistical significance was $P=0.05$.

\section{Results}

Clinical data of the patients

Table 1 summarizes the clinicopathological characteristics of the 101 glioma cases studied. There were 64 (63.4\%) male and $37(36.6 \%)$ female patients and the median age of patients was 35 years old (range 2-69 years old). The majority of patients (70.3\%) had preoperative Karnofsky performance scores (KPS) of 70 or greater. The median follow-up period was 20 months (range from 1 to 75 months).

Vasculogenic mimicry is correlated with tumor grade

Although a PAS-positive pattern was seen in most of the samples, CD34-negative and PAS-positive channels, which 
Table 1 Clinical data and VM in glioma patients

\begin{tabular}{|c|c|c|c|c|c|}
\hline & $n$ & VM positive & VM negative & $\chi^{2}$ & $P$ value \\
\hline \multicolumn{6}{|l|}{ Sex } \\
\hline Female & 37 & 3 & 34 & \multirow[t]{2}{*}{0.606} & \multirow[t]{2}{*}{$0.436^{\mathrm{a}}$} \\
\hline Male & 64 & 10 & 54 & & \\
\hline \multicolumn{6}{|l|}{ Age (years) } \\
\hline$<40$ & 63 & 7 & 56 & \multirow[t]{3}{*}{2.872} & \multirow[t]{3}{*}{$0.220^{\mathrm{b}}$} \\
\hline$\geq 40$ to $>50$ & 10 & 0 & 10 & & \\
\hline$\geq 50$ & 28 & 6 & 22 & & \\
\hline \multicolumn{6}{|l|}{ Karnofsky performance scale } \\
\hline$\geq 70$ & 71 & 8 & 63 & \multirow[t]{2}{*}{0.172} & \multirow[t]{2}{*}{$0.678^{\mathrm{a}}$} \\
\hline$<70$ & 30 & 5 & 25 & & \\
\hline \multicolumn{6}{|l|}{ Preoperative epilepsy } \\
\hline Yes & 25 & 2 & 23 & \multirow[t]{2}{*}{0.244} & \multirow[t]{2}{*}{$0.621^{\mathrm{a}}$} \\
\hline No & 76 & 11 & 65 & & \\
\hline \multicolumn{6}{|l|}{ High intra-cranial pressure } \\
\hline Yes & 60 & 9 & 51 & \multirow[t]{2}{*}{0.597} & \multirow[t]{2}{*}{$0.440^{\mathrm{a}}$} \\
\hline No & 41 & 4 & 37 & & \\
\hline \multicolumn{6}{|l|}{ Tumor grade } \\
\hline WHO I & 6 & 0 & 6 & \multirow[t]{4}{*}{11.442} & \multirow[t]{4}{*}{$0.006^{\mathrm{b}}$} \\
\hline WHO II & 35 & 2 & 33 & & \\
\hline WHO III & 39 & 3 & 36 & & \\
\hline WHO IV & 21 & 8 & 13 & & \\
\hline \multicolumn{6}{|l|}{ Extent of tumor resection } \\
\hline Gross-total/subtotal resection & 82 & 11 & 71 & \multirow[t]{2}{*}{0.363} & \multirow[t]{2}{*}{$0.547^{\mathrm{a}}$} \\
\hline Partial resection & 29 & 2 & 27 & & \\
\hline
\end{tabular}

${ }^{a}$ Pearson $\chi^{2}$ test (asymptotic significance, two-sided)

b Fisher's exact test (two-sided)

were considered as VM, were found in 13 of 101 cases (12.9\%) examined (Fig. 1). The cells composing the channels were negative for CD34 indicating that they were not endothelial or endothelial progenitor cells. The detection of GFAP expression in cells around the VM channels confirmed the cells as astrocytic tumor cells (Fig. 1).

To determine the clinical significance of VM in gliomas, we compared the presence of VM with clinicopathological characteristics of the patients. Vasculogenic mimicry was detected preferentially in high-grade gliomas: 8 of 21 WHO grade IV (38.1\%), 3 of 39 WHO grade III (7.7\%), and 2 of 35 WHO grade II $(5.7 \%)$. In contrast, no VM was found in any WHO grade I gliomas examined. The high-grade (WHO grades III and IV) gliomas had a higher incidence of VM than that of lowgrade (WHO grades I and II) gliomas $(P=0.006)$ (Table 1). No associations were found between the existence of VM and the sex, age, preoperative KPS, preoperative epilepsy of the patients, and extent of tumor resection (Table 1).
Vasculogenic mimicry is associated with COX-2 and MMP-9 expressions

No significant associations were found between VM and Ki-67 labeling index or VEGF staining intensity index (Table 2). Tumor specimens that expressed high levels of COX-2 proteins had VM, whereas tumors (generally lowgrade gliomas) with low or no COX-2 expression had fewer evidence of VM $(P<0.05)$ (Table 2). Significant association was found between the existence of VM and MMP-9 expression $(P<0.05)$ (Table 2$)$.

Vasculogenic mimicry is a predictor of poor prognosis

Using the Kaplan-Meier survival analysis, we compared the survival times between patients with and without VM tumors. In patients with VM-positive gliomas, the median survival time was 15 months (95\% CI, 2-28 months), compared with a median survival time of 38 months (95\% CI, 25-50 months) for patients with VM-negative tumors 
Fig. 1 Representative micrographs of VM in glioma. a PAS-positive (purple-red) zone without $\mathrm{CD} 34$ positive cells indicate VM; some angiogenesis vessels are seen with CD34 stained (brown) cells in the internal lumen of vessels. b A magnification of image (a); erythrocyte shadows are seen in the channels. c The cells composing the channels were negative for CD34 indicating that they were not endothelial or endothelial progenitor cells (VM zone); and CD34-positive and PAS-positive channels are seen in the same view (endothelial vessels). d Cells around the channels are stained positive for GFAP indicating that they are astrocytic tumor cells. Scale bars $50 \mu \mathrm{m}$. (Color figure online)
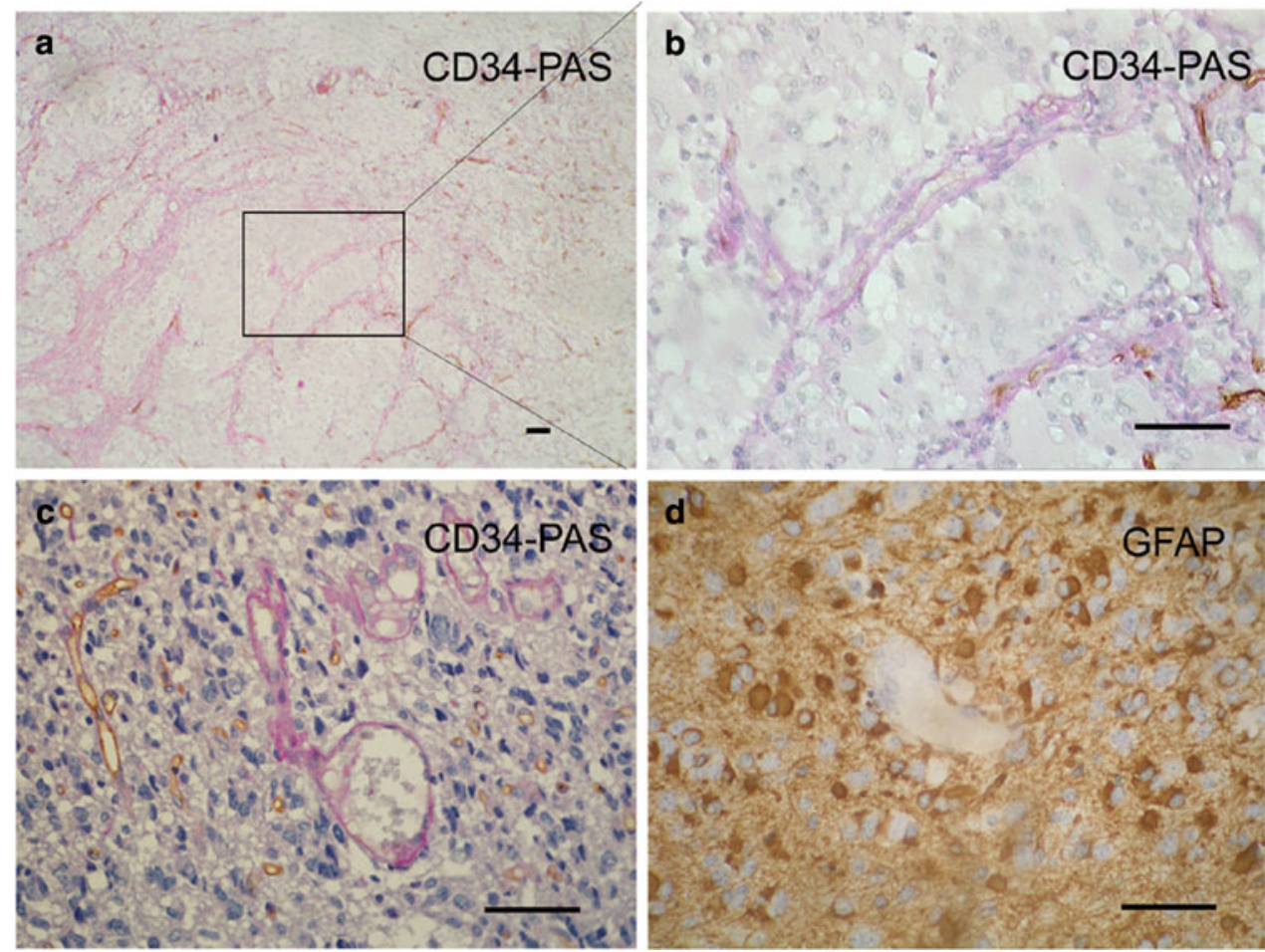

Table 2 Correlation of VM with Ki-67, VEGF, COX-2 and MMP-9 in gliomas

\begin{tabular}{lccll}
\hline & VM positive & VM negative & $Z$ & $P$ value \\
\hline Ki-67 & $1.54 \pm 1.20$ & $1.13 \pm 0.71$ & -1.242 & 0.214 \\
VEGF & $75.47 \pm 74.47$ & $47.50 \pm 60.13$ & -1.474 & 0.141 \\
COX-2 & $87.31 \pm 63.20$ & $43.92 \pm 45.92$ & -2.645 & $0.008^{*}$ \\
MMP-9 & $94.62 \pm 82.09$ & $41.93 \pm 60.79$ & -2.896 & $0.004^{*}$ \\
\hline
\end{tabular}

$* P<0.05$

$(P=0.027)$ (Fig. 2). Since survival in patients with brain tumors has been associated with several clinicopathological variables, we attempted to define the relative contribution of VM expression to survival. Expression of VM correlated significantly with postoperative survival by univariate analysis $(P=0.028)$. The age, KPS, and tumor histology also correlated with survival by univariate analysis. Multivariate analysis and cox proportional hazards model indicated that age and tumor grade were independent predictors of poor prognosis $(P=0.003$ and $P=$ 0.004 , respectively) for glioma patients.

Vasculogenic mimicry is associated with lower MVD in high-grade gliomas

The levels of MVD in low-grade (WHO grades I and II) and high-grade (WHO III and IV) gliomas were $21.41 \pm 18.38$ and $34.10 \pm 32.52$, respectively. However, no significant correlation was found between MVD and postoperative

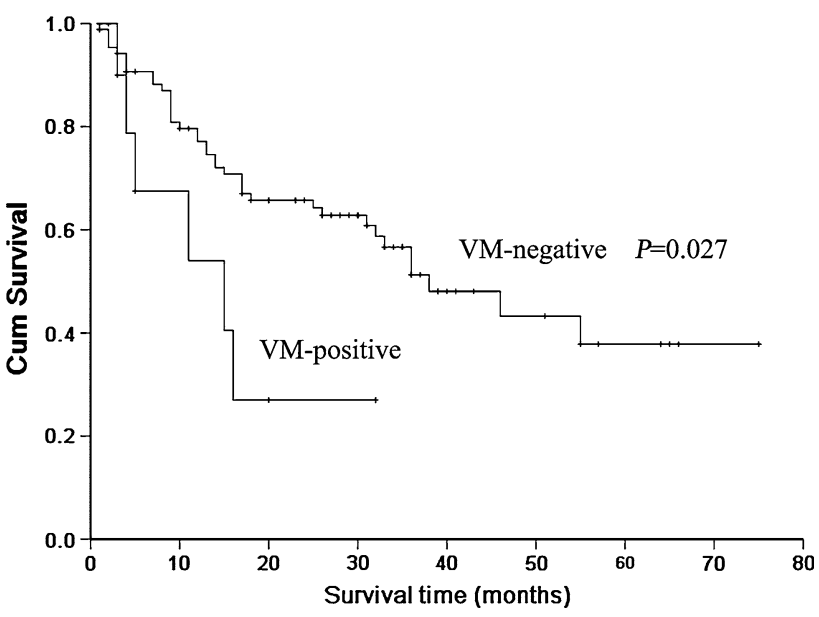

Fig. 2 Patients whose tumors have VM show shorter survival than those without VM $(P=0.027)$ by Kaplan-Meier survival analysis

survival by univariate analysis $(P=0.110)$. We then explored potential association between VM and MVD in high-grade gliomas. Microvascular density was detected at a higher level in VM-negative tumors $(45.17 \pm 38.96)$ than in VM-positive tumors $(21.67 \pm 20.05)$ in high-grade gliomas $(Z=-2.064, P=0.039)$ (Table 3$)$.

\section{Discussion}

For years, sprouting angiogenesis has been considered an exclusive mechanism of tumor vascularization. However, 
Table 3 Correlation of VM with MVD in high-grade gliomas

\begin{tabular}{lllll}
\hline & VM positive & VM negative & $Z$ & $P$ value \\
\hline $\begin{array}{c}\text { MVD } \\
(\text { mean } \pm \mathrm{SD})\end{array}$ & $21.67 \pm 20.05$ & $45.17 \pm 38.96$ & -2.064 & 0.039 \\
\hline
\end{tabular}

over the last decade, several other tumor vascularization mechanisms have been identified, including vessel-cooption, intussusception and recruitment of endothelial precursor cells. One mechanism that does not involve endothelial cells, namely VM, has also been reported. The latter describes a mechanism by which highly aggressive tumor cells form vessel-like structures themselves, by virtue of their high plasticity. According to Maniotis et al. [2], VM channels are patterned networks of interconnected loops of PAS-positive extracellular matrix that may be solid or hollow, with no involvement of endothelial cells but with red blood cells readily detectable inside such channels. The current study investigated the prevalence and clinical significance of VM in gliomas. CD34-negative and PAS-positive channels were detected in $12.9 \%$ of astrocytic tumors examined. The VM channels were arranged in arcs, loops and networks. These results were in line with our previous findings of VM in gliomas [11]. Vasculogenic mimicry was detected predominantly in high-grade gliomas and a significant association was found between VM and tumor grade.

The VEGF stimulates proliferation of endothelial cells through specific tyrosine kinase receptors, flt-1 and flt/KDR, and is a central regulator of the angiogenic process [14, 15]. We performed immunostaining for Ki-67 and VEGF to further evaluate whether VM channels in gliomas were associated with cell proliferation. In this study, no significant associations have been found between VM and expression of Ki-67 or VEGF. These results indicate that VM in gliomas may be distinct from endothelial lined vessels.

The COX-2, inducible isoform of prostaglandin $\mathrm{H}$ synthase, has been implicated in the growth and progression of a variety of human cancers. Over-expression of COX-2 is known to correlate with the aggressive and invasive potential of tumor cells [16]. It has been reported that breast tumor cells over-expressing COX-2 developed vascular channels when plated on three-dimensional matrigel cultures $[17,18]$. We hypothesized that $\mathrm{COX}-2$ may play a role in VM formation. To this end, we evaluated COX-2 expression in gliomas by immunohistochemistry and correlated the results with VM. A significant association was observed between VM and COX-2 expression, suggesting that COX-2 could contribute to VM formation.

Matrix metalloproteinases (MMPs) are enzymes that promote tumor invasion and angiogenesis by enzymatically remodeling the extracellular matrix. MMP-9 is the most abundant form of MMPs in malignant gliomas. Our result revealed a significant association between VM and MMP-9 expression. These results provide evidence that VM may associate with the aggressive and invasive nature of gliomas.

Vasculogenic mimicry channels have been observed in several tumor types and the occurrence is strongly associated with a poor prognosis. Guzman et al. [8] reported that VM of the patterned matrix type was present in hepatocellular carcinoma and was associated with tumor recurrence after orthotopic liver transplantation. Vasculogenic mimicry is also involved in the invasion and metastasis of malignant tumors and has been associated with poor prognosis in malignant esophageal stromal tumors [19], colorectal cancer [20], renal cell carcinoma [21] and sarcomas [6]. However, there has been a study reporting that there was no prognostic impact of VM in pT3 and pT4 cutaneous melanoma [22]. To our knowledge, there have been no reports on whether the presence of VM channels would have an impact on the outcome of glioma patients. In this study, our findings are consistent with the abovementioned results, that patients with VM positive tumors have a shorter survival time than those without VM. However, whether the poor prognosis of patients with VM was due to a high pathological grade requires further exploration. Patients in our study consisted of 60 cases of high-grade gliomas. The Kaplan-Meier survival curve also showed a significant difference for survival times between VM-positive and VM-negative groups $(P=0.008)$. However, when we analyzed the glioblastoma (only 21 cases) group independently, there was no significant difference of survival time between VM-positive and VM-negative groups $(P=0.493)$. This is not surprising, since we have revealed that, although there was no significant correlation between MVD and survival time of the patients $(P=0.110)$, the MVD were much higher in VM-negative than in VM-positive high-grade gliomas $(P=0.039)$. Thus, VM could be a complementation to ensure blood supply, especially in regions of the gliomas with lower MVD. Therefore, the phenomenon of patients with VM positive gliomas having poorer prognosis may not, or at least not only, be due to histological grade of the tumor. Survival of patients with malignant astrocytomas has been associated with several clinicopathological variables including age, KPS of the patients, tumor grade, the extent of surgical resection for tumors and postoperative radiotherapy. Vasculogenic mimicry along with MVD could also be defined as one of the predictors for poor outcome of the glioma patient.

The growth of tumor depends on angiogenesis and microvascular adaptation. It has been well established that the degree of vascularity correlates with increasing malignancy [23] in gliomas. Zhang et al. [24] has proposed a three-stage phenomenon among VM channels, mosaic 
blood vessels, and endothelium-dependent blood vessels, wherein all three patterns participate in tumor blood supply. Vasculogenesis/angiogenesis is the most recognized cellular process that is activated in tumors to fulfill this need. When tumors grow too rapidly, the current vascular structure would not be able to provide sufficient nutrients and oxygen. Since not all high-grade gliomas possess a high microvessel density, we can speculate that other factors may modulate the blood supply in these tumors. Tumor cells may utilize their tremendous plasticity [25-28] to form vascular structures to mimic blood vessels [26] under hypoxic conditions. In other words, when the level of MVD would not be high enough to provide blood to tumors, VM will develop, otherwise tumor necrosis could present. First, while we found that the average level of MVD was higher in high-grade gliomas than in low-grade gliomas, not all the high-grade gliomas had a high level of MVD. Second, although it has been reported $[29,30]$ that the level of microvessels per microscopic field has prognostic significance in astrocytomas, some researchers observed that the level of MVD did not correlate with survival in paediatric astrocytomas by univariate analysis [31]. We have also not observed a significant relationship between MVD and prognosis of the glioma patients in this study. Thus, it is possible that tumor angiogenesis requires the consideration of other types of blood supply. Third, we have identified two clear subgroups of patients based on VM channels in the glioma specimen to analyze the relationship between VM and MVD. Our data showed that MVD was comparably less in VM-positive tumors than in VM-negative tumors in high-grade gliomas. In all, we believe that the existence of VM might provide a complementation to ensure tumor blood supply, and may serve as another mechanism to obtain nutrients for survival, especially in less MVD regions of the gliomas.

Recently, Wang et al. [32] reported that the stem-celllike CD133(+) fraction from glioblastoma included a subset of vascular endothelial-cadherin (CD144)-expressing cells. They suggest that a subpopulation of the CD133(+) cells were multipotent and capable of differentiation along tumor and endothelial lineages. Though the detailed molecular mechanisms for VM are not fully understood, glioma stem cells (GSCs) might play a key role since GSCs have been shown to be involved in tumor tissue remodeling [28]. There has been evidence strongly suggesting that GSCs are capable of forming blood vessels as well as 'tubular vasculogenic mimicry' de novo [27]. There is also evidence of a physiological connection between the endothelial-lined vasculature and the VM channel [28]. In other words, VM is defined by the unique ability of aggressive tumor cells to express an endothelial cell phenotype.

In conclusion, the presence of $\mathrm{VM}$ is associated with higher grade in gliomas, and may correlate with the aggressive and invasive nature of gliomas. The existence of VM may provide a complementation to ensure blood supply, especially in less vascularized regions of the tumor. Our data suggest that gliomas with VM-positive networks may identify patients with poorer prognosis.

Acknowledgment This study was supported by a grant from the National Nature Science Foundation of China (No. 30571914).

Conflict of interest The authors report no conflict of interest concerning the materials or methods used in this study or the findings specified in this paper.

Open Access This article is distributed under the terms of the Creative Commons Attribution Noncommercial License which permits any noncommercial use, distribution, and reproduction in any medium, provided the original author(s) and source are credited.

\section{References}

1. Shrieve DC, Alexander E 3rd, Black PM et al (1999) Treatment of patients with primary glioblastoma multiforme with standard postoperative radiotherapy and radiosurgical boost: prognostic factors and long-term outcome. J Neurosurg 90:72-77

2. Maniotis AJ, Folberg R, Hess A et al (1999) Vascular channel formation by human melanoma cells in vivo and in vitro: vasculogenic mimicry. Am J Pathol 155:739-752

3. Sharma N, Seftor RE, Seftor EA et al (2002) Prostatic tumor cell plasticity involves cooperative interactions of distinct phenotypic subpopulations: role in vasculogenic mimicry. Prostate 50:189-201

4. Shirakawa K, Kobayashi H, Heike Y et al (2002) Hemodynamics in vasculogenic mimicry and angiogenesis of inflammatory breast cancer xenograft. Cancer Res 62:560-566

5. Sood AK, Fletcher MS, Zahn CM et al (2002) The clinical significance of tumor cell-lined vasculature in ovarian carcinoma: implications for anti-vasculogenic therapy. Cancer Biol Ther $1: 661-664$

6. Sun B, Zhang S, Zhao X et al (2004) Vasculogenic mimicry is associated with poor survival in patients with mesothelial sarcomas and alveolar rhabdomyosarcomas. Int J Oncol 25:1609-1614

7. Cai XS, Jia YW, Mei J et al (2004) Tumor blood vessels formation in osteosarcoma: vasculogenesis mimicry. Chin Med J (Engl) 117:94-98

8. Guzman G, Cotler SJ, Lin AY et al (2007) A pilot study of vasculogenic mimicry immunohistochemical expression in hepatocellular carcinoma. Arch Pathol Lab Med 131:1776-1781

9. Sun B, Qie S, Zhang S et al (2008) Role and mechanism of vasculogenic mimicry in gastrointestinal stromal tumors. Hum Pathol 39:444-451

10. Folberg R, Hendrix MJ, Maniotis AJ (2000) Vasculogenic mimicry and tumor angiogenesis. Am J Pathol 156:361-381

11. Yue WY, Chen ZP (2005) Does vasculogenic mimicry exist in astrocytoma? J Histochem Cytochem 53:997-1002

12. Louis DN, Ohgaki H, Wiestler OD et al (2007) The 2007 WHO classification of tumours of the central nervous system. Acta Neuropathol 114:97-109

13. Krishna M, Smith TW, Recht LD (1995) Expression of bcl-2 in reactive and neoplastic astrocytes: lack of correlation with presence or degree of malignancy. J Neurosurg 83:1017-1022

14. Matsuo M, Sakurai H, Saiki I (2003) ZD1839, a selective epidermal growth factor receptor tyrosine kinase inhibitor, shows 
antimetastatic activity using a hepatocellular carcinoma model. Mol Cancer Ther 2:557-561

15. Fox SB, Turley H, Cheale M et al (2004) Phosphorylated KDR is expressed in the neoplastic and stromal elements of human renal tumours and shuttles from cell membrane to nucleus. J Pathol 202:313-320

16. Rozic JG, Chakraborty C, Lala PK (2001) Cyclooxygenase inhibitors retard murine mammary tumor progression by reducing tumor cell migration, invasiveness and angiogenesis. Int J Cancer 93:497-506

17. Basu GD, Liang WS, Stephan DA et al (2006) A novel role for cyclooxygenase- 2 in regulating vascular channel formation by human breast cancer cells. Breast Cancer Res 8:R69

18. Basu GD, Pathangey LB, Tinder TL et al (2005) Mechanisms underlying the growth inhibitory effects of the cyclo-oxygenase-2 inhibitor celecoxib in human breast cancer cells. Breast Cancer Res 7:R422-R435

19. Zhao H, Gu XM (2008) Study on vasculogenic mimicry in malignant esophageal stromal tumors. World J Gastroenterol 14: 2430-2433

20. Baeten CI, Hillen F, Pauwels P et al (2009) Prognostic role of vasculogenic mimicry in colorectal cancer. Dis Colon Rectum 52:2028-2035

21. Vartanian AA, Stepanova EV, Gutorov SL et al (2009) Prognostic significance of periodic acid-Schiff-positive patterns in clear cell renal cell carcinoma. Can J Urol 16:4726-4732

22. Massi D, Franchi A, Paglierani M et al (2004) Vasculogenic mimicry has no prognostic significance in pT3 and pT4 cutaneous melanoma. Hum Pathol 35:496-502

23. Kohlberger PD, Obermair A, Sliutz G et al (1996) Quantitative immunohistochemistry of factor VIII-related antigen in breast carcinoma: a comparison of computer-assisted image analysis with established counting methods. Am J Clin Pathol 105:705-710

24. Zhang S, Guo H, Zhang D et al (2006) Microcirculation patterns in different stages of melanoma growth. Oncol Rep 15:15-20

25. Hendrix MJ, Seftor EA, Hess AR et al (2003) Vasculogenic mimicry and tumour-cell plasticity: lessons from melanoma. Nat Rev Cancer 3:411-421

26. Mirshahi P, Rafii A, Vincent L et al (2009) Vasculogenic mimicry of acute leukemic bone marrow stromal cells. Leukemia 23:1039-1048

27. El Hallani S, Boisselier B, Peglion F et al (2010) A new alternative mechanism in glioblastoma vascularization: tubular vasculogenic mimicry. Brain 133:973-982

28. Dong J, Zhang Q, Huang Q et al (2010) Glioma stem cells involved in tumor tissue remodeling in a xenograft model. J Neurosurg 113:249-260

29. Abdulrauf SI, Edvardsen K, Ho KL et al (1998) Vascular endothelial growth factor expression and vascular density as prognostic markers of survival in patients with low-grade astrocytoma. J Neurosurg 88:513-520

30. Leon SP, Folkerth RD, Black PM (1996) Microvessel density is a prognostic indicator for patients with astroglial brain tumors. Cancer 77:362-372

31. Birlik B, Canda S, Ozer E (2006) Tumour vascularity is of prognostic significance in adult, but not paediatric astrocytomas. Neuropathol Appl Neurobiol 32:532-538

32. Wang R, Chadalavada K, Wilshire J et al (2010) Glioblastoma stem-like cells give rise to tumour endothelium. Nature 468: 829-833 